\title{
Obstructive sleep apnoea in patients undergoing cardiac surgery: Screening, postoperative outcomes and perioperative care
}

Martina Mason*, Ian Smith

Papworth Hospital NHS Foundation Trust, Papworth Everard, Cambridge, CB23 3RE, UK

\section{Article Info}

\section{Article Notes}

Received: December 20, 2017

Accepted: January 23, 2018

\section{*Correspondence:}

Dr. Martina Mason, Papworth Hospital NHS Foundation

Trust, Papworth Everard, Cambridge, CB23 3RE, UK;

Tel. No: +44 (0)1480 364172; Fax. number: 01480364161

E-mail: martina.mason@papworth.nhs.uk

C 2018 Mason M. This article is distributed under the terms of the Creative Commons Attribution 4.0 International License.

\section{Keywords}

Sleep apnoea

Screening

Cardiac surgery

Postoperative outcomes

\begin{abstract}
Obstructive sleep apnoea (OSA) is common and high prevalence has been described amongst patients undergoing cardiac revascularisation surgery. An excess of postoperative complications has been reported in patients with untreated Obstructive Sleep Apnoea (OSA) following surgical procedures, including those undergoing cardiac surgery. This has led some clinicians towards pre-operative screening for OSA though the best screening methodology has not yet been established. Moreover, the effect of screening and of treatment for OSA on surgical outcomes remains unknown. Does current evidence justify screening and treating patients before they present for surgery? Is this leading to potential delay in surgery whilst awaiting sleep diagnostics and commencing the treatment? This review article will examine the available evidence base and endeavour to answer these questions and identify implications for future research.
\end{abstract}

\section{OSA and cardiovascular disease}

Sleep Disordered Breathing is a term used to cover a range of breathing events encountered during sleep and includes obstructive sleep apnoea (OSA), central sleep apnoea, Cheyne-Stokes respiration and respiratory effort related arousals. OSA is the most common form of sleep disordered breathing. OSA is common ${ }^{1}$ and the reported prevalence has increased in the last two decades ${ }^{1}$ with the new data reported mainly in European population of adults 4085 years old showing that at least mild OSA (apnoea-hypopnoea index $\geq 5$ ) was present in $84 \%$ of men and $61 \%$ of women and at least moderate disease (apnoea-hypopnoea index $\geq 15$ ) was present in $50 \%$ of men and $23 \%$ of women ${ }^{2}$. OSA and cardiac disease share some common risk factors and in patients undergoing coronary artery bypass graft (CABG) procedures reported prevalence is high, ranging between 41 and $87 \% \%^{3-5}$. High proportions of patients may present for surgery without a previous diagnosis of OSA ${ }^{6,7}$ and may be at risk of worse postoperative outcomes ${ }^{8}$. In patients with moderate to severe OSA syndrome Continuous Positive Airway Pressure (CPAP) is the conventional treatment with established clinical and cost effectiveness ${ }^{9}$ but its effect on cardiovascular outcomes has not been confirmed. A recent, large, randomised controlled trial of adults with moderate to severe OSA and pre-existing cardiovascular disease showed that the use of CPAP had no significant effect on the prevention of recurrent serious cardiovascular events ${ }^{10}$. It is possible that patients with compliance exceeding $4 \mathrm{~h} / \mathrm{night}$ may 
benefit in terms of cardiac outcomes but further research is needed to clarify this benefit ${ }^{11,12}$.

\section{Postoperative outcomes in patients with OSA undergoing cardiac surgery}

There is evidence that patients with untreated OSA, compared to those without this diagnosis, undergoing general surgical procedures, have worse postoperative outcomes including increased risks of post-operative cardiac events, acute respiratory failure and ICU transfers reported in two meta-analyses ${ }^{13,14}$. Data in patients undergoing cardiac surgery are inconsistent with some results suggesting an association between OSA and postoperative complications and others not supporting this association ${ }^{15,16}$. A study of 67 prospectively recruited participants, assessed for OSA by polysomnography (PSG) showed no association between OSA and short term complications but reported that the OSA was independently associated with a higher rate of long-term cardiovascular events after $\mathrm{CABG}^{15}$. Another study by Foldvary-Schaefer et al. recruited prospectively 107 patients and shown that OSA was not significantly associated with worse postoperative outcomes but authors commented that a small study sample could have influenced results ${ }^{16}$.

A recent meta-analysis of 11 studies showed higher odds of adverse cardiac and cerebrovascular events with an odds ratio (OR) of $2.4 ; 95 \% \mathrm{CI} 1.38-4.2 ; \mathrm{p}=0.002$ as well as newly documented postoperative atrial fibrillation (POAF), OR = 1.94; CI 1.13-3.33; $\mathrm{p}=0.02$ in patients with OSA compared to non OSA patients. ${ }^{8}$. It included 11 studies and showed higher odds of adverse cardiac and cerebrovascular events with an odds ratio (OR) of 2.4; $95 \%$ CI 1.38-4.2; $\mathrm{p}=0.002$ as well as newly documented postoperative atrial fibrillation (POAF), OR $=1.94$; CI 1.133.33; $p=0.02$ in patients with OSA compared to non OSA patients $^{8}$. The majority of patients in this meta-analysis were newly diagnosed, untreated patients with OSA. The studies included were of mixed quality. Only five out of 11 of the studies were prospective and used recognised sleep diagnostic (PSG or watch-PAT) to diagnose OSA ${ }^{15-19}$ whilst the rest were either retrospective cohort assessments or used questionnaires to examine for high risk of OSA. Out of the five higher quality studies, two report no association between OSA and postoperative outcomes ${ }^{15,16}$, two report an association between OSA and POAF ${ }^{17,19}$ and one with prolonged ventilation, tracheostomy, Intensive Care Unit and hospital stay ${ }^{18}$. The fact that the definitions of hypopnea have changed several times over the years, and that many of the studies included in the meta-analyses do not give specifics about their diagnostic procedures, may be a reason why studies reach different conclusions.

We have recently reported outcomes on 122 prospectively recruited patients undergoing $C A B G$ and $C A B G$ and valve surgery in a Tertiary cardiothoracic centre. Patients underwent inpatient overnight oximetry on the night prior to their surgery to assess for the presence of sleep apnoea which was diagnosed in patients with an arterial oxygen desaturation index (ODI) $\geq 5 / \mathrm{hr}$. The primary outcome measure was length of stay (LoS) in ICU in days and the secondary outcome a composite measure of post-operative complications in ICU. We found a high prevalence of newly diagnosed sleep apnoea (47\%) in our cohort as well as an association of increasing severity of sleep apnoea with postoperative complications, in particular cardiovascular and renal, including acute kidney injury, heart failure and hypotension. This adds to the evidence for a positive association between sleep apnoea and adverse postoperative outcomes in this surgical cohort. Our study was the first to report an association between severity of sleep apnoea and adverse postoperative outcomes in a cardiac surgical cohort, where interestingly, even patients with ODI 5-15/ $\mathrm{hr}$, often regarded as clinically insignificant, had a higher probability of developing post-operative complications than patients with no desaturations overnight preoperatively ${ }^{4}$. An association between sleep apnoea and postoperative acute kidney injury was also reported in a recent study in multi-ethnic Asian population. OSA with the repetitive nocturnal arousals causes sympathetic nervous system activation which persists during the day ${ }^{20,21}$ and along with fluid imbalances during and after surgery could contribute to hypoperfusion of the renal arteries and development of kidney injury.

In our study we could not distinguish between OSA and central sleep apnoea as our diagnostic was nocturnal oximetry. It is possible that central sleep apnoea, common in patients with heart failure and ischaemic heart disease $e^{22}, 23$ contributed to significant respiratory events identified on overnight sleep monitoring. Central sleep apnoea is characterised by pauses in breathing with no apparent occlusion of the airway and there are no published data on its impact on surgical outcomes though it has been implicated in adverse outcomes for patients with cardiac disease ${ }^{24}$. Further research is needed to assess its impact on postoperative outcomes in patients undergoing surgical procedures.

In keeping with the outcome from one recent metaanalysis ${ }^{8}$, we did not find a significant association between OSA and Intensive Care Unit length of stay. It is possible that the lack of such an association is due to the small sample size of individual studies and complexity of the process of Intensive Care Unit discharge, which can depend on local policies and the availability of beds on the receiving ward over and above the clinical condition of the patient.

\section{Effect of Continuous Positive Airway Pressure on postoperative outcomes}

It is apparent, from the presented evidence, that OSA 
is common in cardiac surgical population and that it is associated with worse postoperative complications ${ }^{4,8,25}$. Although Continuous Positive Airway Pressure (CPAP) is the conventional treatment for patients with OSA syndrome with established clinical and cost effectiveness ${ }^{9}$ its effect on patients with OSA in perioperative settings has yet to be demonstrated. A reduction in pulmonary complications, in patients without a diagnosis of OSA, undergoing thoracoabdominal and cardiac surgery has been reported ${ }^{26,27}$ but the evidence supporting the application of perioperative CPAP to patients with OSA is currently lacking. A recent meta-analysis of 6 studies including 904 patients showed no significant difference in post-operative adverse events between CPAP and non-CPAP treatment groups in patients undergoing general surgery ${ }^{28}$. There is some evidence for improvement in cardiovascular morbidity in a retrospective cohort of 26,842 patients with OSA undergoing vascular surgery where those not treated with positive airway pressure preoperatively were at increased risks for cardiopulmonary complications including unplanned reintubations (OR 2.5) and myocardial infarction (OR 2.6) compared with treated OSA patients ${ }^{29}$. The effect of CPAP on patients with OSA undergoing cardiac surgery has not been examined in randomized controlled trials.

\section{Implications for practice and future direction}

Current evidence from observational studies suggests increased risks of adverse cardiac, cerebrovascular events as well as POAF in patients with untreated OSA undergoing cardiac surgical procedures. In addition two prospective observational studies also report association between sleep apnoea and adverse renal outcomes. The effect of central sleep apnoea on postoperative outcomes in cardiac surgical cohort remains unknown and further research is needed in this area. There is no evidence at present that confirms that early awareness and treatment of OSA with CPAP improves perioperative outcomes and further research is necessary to clarify the costs and benefits of preoperative diagnosis and treatment of OSA. Until then a pragmatic approach in the pre-assessment setting should be adopted focusing mainly on a detailed clinical history. In addition, various screening tools, including questionnaires, have been developed and validated in different surgical cohorts to identify patients at high risk of OSA prior to surgery. In systematic reviews of screening questionnaires for OSA, the STOP-Bang questionnaire had the highest methodological validity, moderately high sensitivity and best negative predictive value for assessing the risk of moderate/severe OSA and could be used to help clinician to identify patients at high risk for OSA in pre-assessment clinics $\frac{30,31}{}$, with prompt onward referral of those with suspected OSA syndrome to avoid unnecessary delays in surgery.

\section{References}

1. Peppard PE, Young T, Barnet JH, et al. Increased prevalence of sleepdisordered breathing in adults. American journal of epidemiology. 2013 ;177(9): 1006-14.

2. Heinzer R, Vat S, Marques-Vidal P, et al. Prevalence of sleep-disordered breathing in the general population: the HypnoLaus study. The Lancet Respiratory medicine. 2015; 3(4): 310-8.

3. Amra B, Niknam N, Sadeghi MM, et al. Obstructive sleep apnea and postoperative complications in patients undergoing coronary artery bypass graft surgery: a need for preventive strategies. International journal of preventive medicine. 2014; 5(11): 1446-51.

4. Mason M, Hernandez Sanchez J, Vuylsteke A, et al. Association between severity of untreated sleep apnoea and postoperative complications following major cardiac surgery: a prospective observational cohort study. Sleep Med. 2017; 37: 141-46.

5. Danzi-Soares J, Genta PR, Nerbass FB, et al. Obstructive sleep apnea is common among patients referred for coronary artery bypass grafting and can be diagnosed by portable monitoring. Coronary Artery Disease. 2012; 23(1): 31-8.

6. Young T, Evans L, Finn L, et al. Estimation of the clinically diagnosed proportion of sleep apnea syndrome in middle-aged men and women. Sleep. 1997; 20(9): 705-6.

7. Singh M, Liao P, Kobah S, et al. Proportion of surgical patients with undiagnosed obstructive sleep apnoea. Br J Anaesth. 2013; 110(4): 629-36.

8. Nagappa M, Ho G, Patra J, et al. Postoperative Outcomes in Obstructive Sleep Apnea Patients Undergoing Cardiac Surgery: A Systematic Review and Meta-analysis of Comparative Studies. Anesth Analg. 2017; 125(6): 2030-37.

9. Giles TL, Lasserson TJ, Smith BH, et al. Continuous positive airways pressure for obstructive sleep apnoea in adults. Cochrane Database Syst Rev. 2006(3): CD001106.

10. McEvoy RD, Antic NA, Heeley E, et al. CPAP for Prevention of Cardiovascular Events in Obstructive Sleep Apnea. The New England journal of medicine. 2016; 375(10): 919-31.

11. Khan SU, Duran CA, Rahman H, et al. A meta-analysis of continuous positive airway pressure therapy in prevention of cardiovascular events in patients with obstructive sleep apnoea. European heart journal. 2017.

12. Abuzaid AS, Al Ashry HS, Elbadawi A, et al. Meta-Analysis of Cardiovascular Outcomes With Continuous Positive Airway Pressure Therapy in Patients With Obstructive Sleep Apnea. The American journal of cardiology. 2017; 120(4): 693-99.

13. Kaw R, Chung F, Pasupuleti V, et al. Meta-analysis of the association between obstructive sleep apnoea and postoperative outcome. Br J Anaesth. 2012; 109(6): 897-906.

14. Hai F, Porhomayon J, Vermont L, et al. Postoperative complications in patients with obstructive sleep apnea: a meta-analysis. J Clin Anesth. 2014; 26(8): 591-600.

15. Uchoa CH, Danzi-Soares Nde J, Nunes FS, et al. Impact of OSA on cardiovascular events after coronary artery bypass surgery. Chest. $2015 ; 147(5): 1352-60$.

16. Foldvary-Schaefer N, Kaw R, Collop N, et al. Prevalence of Undetected Sleep Apnea in Patients Undergoing Cardiovascular Surgery and Impact on Postoperative Outcomes. Journal of clinical sleep medicine : JCSM : official publication of the American Academy of Sleep Medicine. 2015; 11(10): 1083-9.

17. Mooe T, Gullsby S, Rabben T, et al. Sleep-disordered breathing: a novel predictor of atrial fibrillation after coronary artery bypass surgery. Coron Artery Dis. 1996; 7(6): 475-8. 
18. Bhama JK, Spagnolo S, Alexander EP, et al. Coronary revascularization in patients with obstructive sleep apnea syndrome. Heart Surg Forum. 2006; 9(6): E813-7.

19. Zhao LP, Kofidis T, Lim TW, et al. Sleep apnea is associated with newonset atrial fibrillation after coronary artery bypass grafting. Journal of critical care. $2015 ; 30(6)$ : 1418 e1-5.

20. Narkiewicz K, Somers VK. Sympathetic nerve activity in obstructive sleep apnoea. Acta physiologica Scandinavica. 2003; 177(3): 385-90.

21. Somers VK, Mark AL, Zavala DC,et al. Contrasting effects of hypoxia and hypercapnia on ventilation and sympathetic activity in humans. J Appl Physiol. (1985) 1989; 67(5): 2101-6.

22. Moruzzi P, Sarzi-Braga S, Rossi M, et al. Sleep apnoea in ischaemic heart disease: differences between acute and chronic coronary syndromes. Heart. 1999; 82(3): 343-7.

23. Javaheri S, Parker TJ, Liming JD, et al. Sleep apnea in 81 ambulatory male patients with stable heart failure. Types and their prevalences, consequences, and presentations. Circulation. 1998; 97(21): 2154-9.

24. Aurora RN, Chowdhuri S, Ramar K, et al. The treatment of central sleep apnea syndromes in adults: practice parameters with an evidence-based literature review and meta-analyses. Sleep. 2012; 35(1): 17-40.

25. Kua J, Zhao LP, Kofidis T, et al. Sleep apnoea is a risk factor for acute kidney injury after coronary artery bypass grafting. European journal of cardio-thoracic surgery : official journal of the European Association for Cardio-thoracic Surgery. 2016; 49(4): 1188-94.

26. Kindgen-Milles D, Muller E, Buhl R, et al. Nasal-continuous positive airway pressure reduces pulmonary morbidity and length of hospital stay following thoracoabdominal aortic surgery. Chest. 2005; 128(2): 821-8.

27. Zarbock A, Mueller E, Netzer S, et al. Prophylactic nasal continuous positive airway pressure following cardiac surgery protects from postoperative pulmonary complications: a prospective, randomized, controlled trial in 500 patients. Chest. 2009; 135(5): 1252-59.

28. Nagappa M, Mokhlesi B, Wong J, et al. The Effects of Continuous Positive Airway Pressure on Postoperative Outcomes in Obstructive Sleep Apnea Patients Undergoing Surgery: A Systematic Review and Meta-analysis. Anesth Analg. 2015; 120(5): 1013-23.

29. Abdelsattar ZM, Hendren S, Wong SL, et al. The Impact of Untreated Obstructive Sleep Apnea on Cardiopulmonary Complications in General and Vascular Surgery: A Cohort Study. Sleep. 2015; 38(8): 1205-10.

30. Chiu HY, Chen PY, Chuang LP, et al. Diagnostic accuracy of the Berlin questionnaire, STOP-BANG, STOP, and Epworth sleepiness scale in detecting obstructive sleep apnea: A bivariate meta-analysis. Sleep Med Rev. 2017; 36: 57-70.

31. Abrishami A, Khajehdehi A, Chung F. A systematic review of screening questionnaires for obstructive sleep apnea. Can J Anaesth. 2010; 57(5): 423-38. 